CONTINUOUS TRUTH I

Non-constructive Objects

Michael P. Fourman

Department of Mathematics

Columbia University

New York, N.Y. 10027 U.S.A.
Department of Pure Mathematics University of Sydney

N.S.W. 2006

Austra] ia

\begin{abstract}
We give a general theory of the logic of potentially infinite objects, derived from a theory of meaning for statements concerning these objects. The paper has two main parts which may be read independently but are intended to complement each other. The first part is essentially philosophical. In it, we discuss the theory of meaning. We believe that even the staunchest realist must view potential infinities operationally. The second part is formal. In it, we consider the interpretation of logic in the gros topos of sheaves over the category of separable locales equipped with the open cover topology. We show that general principles of continuity, local choice and local compactness hold for these models. We conclude with a brief discussion of the philosophical significance of our formal results. They allow us to reconcile our explanation of meaning with the "equivalence thesis", that rsnow is white is true ' iff snow is white.
\end{abstract}

\title{
PROLEGOMENON
}

Classical mathematics is based on a platonic view of mathematical objects. The meanings of mathematical statements are determined truth-functionally. This Fregean explanation of meaning justifies classical logic. The deficiencies of such a view are amply discussed by Dummett [1978].

A constructive mathematician rejects the completed infinities of classical mathematics. For him, the objects of mathematics are essentially finite. The meaning of quantification over infinite domains is given operationally in terms of a theory of constructions. The resulting logic includes Heyting's predicate calculus and other principles (e.g. choice principles).

As Dummett has stressed, one task of any philosophy of mathematics is to explain the applicability of mathematics. The potential infinities of experience exceed the finite objects of the strict constructivist. They demand a mathematics of infinite objects. Naive abstraction leads to the ideal infinite objects of classical mathematics. This idealisation has enjoyed remarkable success. However, the meaning of statements of classical mathematics remains problematic.

Brouwer [1981] introduced to mathematics potentially infinite objects such as freechoice sequences. Consideration of these justified, for Brouwer, intuitionistic logic, including various choice and continuity principles. We shall consider a general notion of non-constructive object. For us, to present such a notion is to give a theory of meaning for statements involving non-constructive objects.

Our non-constructive objects are not the platonic ideal objects of classical mathematics nor the finitary objects of pure constructivism. They are potentially 
infinite objects related to the lawless sequences of Kreisel [1968] and to Brouwer's free-choice sequences (Troelstra [1977]). The meanings of statements about these objects cannot be given in terms of truth conditions (as for classical platonist mathematics) or in terms of constructions (as for naive constructivism). The essence of these non-constructive objects lies in their infinite character. They are not, in general, totally grasped. They are given in terms of partial data which may later be refined. Meaning for statements about non-constructive objects is given by saying what data justifies a given assertion.

To describe a particular notion of non-constructive object is to describe the type of data on which it is based. We consider various such notions. Each conception of data gives an explanation of meaning which extends the range of meaningful statements and may be viewed as introducing new objects in that it ascribes meaning to new forms of quantification. In fact for each type of data we introduce a concrete representation of the non-constructive objects based on it.

Such a project is not novel: Beth [1947] introduced his models to provide just such an explanation of meaning for choice sequences. Our models generalise Beth's. Dummett [1977] makes a lengthy critique of the view that the intended meanings of of the logical constants are faithfully represented on Beth trees. Since our models generalise Beth's they appear prima facie to be susceptible to the same criticisms. However, Dummett's remarks on the (non)-consonance of the intended meanings of the connectives with their interpretation in Beth trees are directed at a different problem from the one we address. Dummett appears to have overlooked the possibility of separating the problem of explaining the constructive meaning of statements concerning lawi ike objects from that of explaining the intuitionistic meaning of statements concerning choice sequences. Although we know of no satisfactory explanation of constructive truth (in particular, we agree with Dummett that Beth models do not give one), such a separation appears natural. It is possible to conceive of constructive truth independently of choice sequences. Given such a conception, Beth models provide an account of the introduction of non-lawlike objects. It is this type of account we have generalised. By way of example we now consider two notions of data closely related to Beth models. They both arise from the same informal picture.

Imagine receiving from Mars an infinite sequence $\alpha$ of natural numbers. The picture is of a ticker-tape which produces an indefinitely continued finite initial segment a of the sequence $\alpha$. (We write $\alpha \in$ a to mean that a is an initia) segment of $\alpha$.) We want to examine the consequences of treating such undetermined sequences seriously as sequences. (Later we shall introduce more interesting examples.)

A naive view of this example considers the stages by which information arises: at any stage, the possible future data is represented by the collection $N^{<N}$ of all finite sequences. At the next stage one of the sequences $<n>$ (where $n \in N$ ) must appear. Not only do we not yet know which of these possibilities will occur, it is not yet determined which will occur. On the basis of this data we may construct many sequences. The simplest of these, $\alpha$ is given by transcribing the data as it arrives. Thus on the basis of data $b \leq a$, we are justified in asserting that $a$ is an initial segment of $\alpha$. We write this $b \Vdash \alpha \in a$. (We order sequences by setting $b \leq a$ if $a$ is an initial seqment of $b$ since then $b$ allows fewer possibilities for $\alpha$.) Another sequence $\beta$ is given by first writing down a fixed finite sequence $b$ and then continuing with the incoming data. Schematically, $a \Vdash \beta \in b^{\star} a$ where * denotes concatenation, and hence "obviously", for any $c \leq a$, we have $c \Vdash \beta \in b^{\star} a$. We want to make all such "obvious" assumptions about the nature of justification explicit; so we give its two basic structural properties. Justification should be

persistent $\frac{\mathrm{a} \Vdash \phi}{\mathrm{a}^{\star} \mathrm{b} \Vdash \phi}$ 
and

induetive

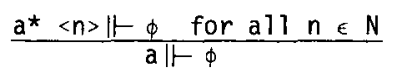

Persistence reflects the idea that knowledge, once justified, is secure. The inductive clause comes from reflection on the infinite character of $\alpha$. Given $\alpha \in a$, the collection $\left\{a^{*}\langle n\rangle \mid n \in N\right\}$ covers all possibilities for future data.

In general, if we stipulate $b \| \phi$ for $b \in B \subseteq N^{<N}$, the principles of induction and persistence will entail other instances $a \| \bar{\phi}$. We call the set $\{a|a| t \phi\}$ the inductive closure of $B$ and say $B$ covers each such $a$.

To describe a particular non-constructive object $y$ is to say what information about it is justified on the basis of each possible item of future data. For example, any monotone function $g: N^{<N} \rightarrow N^{<N}$ (that is $a \leq b$ implies $g(a) \leq g(b)$ ) gives a non-constructive sequence $\gamma$ with a|F $\gamma \in g(a)$ (together with the consequences entailed by the principles of persistence and induction). Monotonicity here embodies the requirement that we should accumulate information on $\gamma$. More generally, we can view any poset $P$ as an abstract representation of data about some type of object. Then a monotone map $\mathrm{N}^{<N} \rightarrow \mathrm{P}$ will represent a non-constructive object of this type.

We do not wish to introduce technicalities here. Later we shall give a definition of $a \| \phi$, for first-order $\phi$, by induction on the structure of $\phi$. For the moment we just remark that such a definition of a $\Vdash \phi$ can be given and that the interpretations of the connectives are completely determined, in the context of our requirements on justification, by requiring that the rules of positive logic be valid. In our present case this would amount to giving Beth's semantics for intuitionistic logic with a slightly modified notion of "bar". Beth's semantics are well known to be equivalent to the topological interpretation over Baire space exploited by Scott [1968] and Moschovakis [1973]; our modification replaces Baire space by formal Baire space (Fourman and Grayson [1982]).

We now return to our picture of the ticker-tape. What we have done is to give a representation of the subjective experience of receiving word from Mars, an external view of how the world will look when data a appears on the ticker-tape. This view is dependent on the contingencies of what data is available. But mathematics should be timeless and absolute. This requirement appears to exclude consideration of potentially infinite objects. We now attempt to resolve this contradiction.

Picture a room with a ticker-tape, $A$ and various sequences in progress, $-\alpha, \beta, \gamma$ described above for example. Now suppose that on the tape we have the finite sequence $b$. We have duly noted that $\alpha \in b, B \in b^{\star} b, \gamma \in g(b)$. Consider now another room A' with a ticker-tape which, as yet, is blank and three non-constructive objects defined by

$$
\begin{aligned}
& c \Vdash-\alpha^{\prime} \in b^{\star} c \\
& c \Vdash-\beta^{\prime} \in b^{*} b^{*} c \\
& c \Vdash-\gamma^{\prime} \in g\left(b^{*} c\right) .
\end{aligned}
$$

In this room on the basis. of no data we can already note that $\alpha^{\prime} \in b, \beta^{\prime} \epsilon b^{\star} b$, $\gamma^{\prime} \in g(b)$. Furthermore data $b^{*} c$ arriving in room $A$ will always have the same consequences for $\alpha, B, \gamma$ as data $c$ arriving in room $A^{\prime}$ has for $\alpha^{\prime}, B^{\prime}, \gamma^{\prime}$. In fact the mathematics (and logic) of the two rooms, A with data $b$ and $A^{\prime}$ with no data, should be the same. We want to add this to our formal treatment. This is done by regarding incoming data not as changing the world but rather as effecting a transformation which changes our view of the world. We consider not a particular ticker-tape but rather the uses which could be made of such an indeterminate sequence to generate non-constructive objects. Data just becomes a way of trans- 
forming one such process into another, generally less free: its restriction.

We give a general definition of this transformation as follows:

$$
c \| \downarrow(\delta \mid b) \text { iff } b^{\star} c \| \phi(\delta)
$$

(Where $\delta$ is a non-constructive object given by stipulating what data justifies $\phi(\delta)$ for various $\left.\phi_{.}\right)$For example, $\alpha^{\prime}\left|b=\alpha^{\prime}=\beta ; \beta\right| b=\beta^{\prime} ; \gamma^{\prime} \mid b=\gamma^{\prime}$.

This change of viewpoint amounts formally to a change in our representation of data. Formerly we considered the partially ordered set or tree $\mathrm{N}^{<N}$ as representing various possible states of information. Incoming data changes the world in that it places us in a new state. Now we consider $N^{<N}$ as a collection of transformations which act to change our view of the world.

Formally it is convenient to represent the data as the monoid of finite sequences under concatenation; if $g: N^{<N} \rightarrow P$ represents a non-constructive object $\gamma$ then $\gamma \mid b$ is represented by $g \circ b$ where $b: a \mapsto b * a$ acts by left concatenation. The

notion of justification is to be stable under such a change of perspective:

$$
a\|-\phi\| b \quad \text { iff } \quad b * a \| \phi
$$

(where $1 b$ is applied to the non-constructive parameters of $\phi_{.}$)

We now consider examples of a more general type of non-constructive object intended to represent potential infinities of experience. We base our description, for the sake of exposition, on a view of classical experimental physics which we ascribe to the nineteenth century. Briefly it runs as follows: Physics is based on measurement. Experiments determine values of parameters a to a certain degree of precision. Generally some error is inevitable but it may in principle be made arbitrarily small (this is the assumption which leads us to labe? this as a nineteenth century notion).

Now, we refuse to admit the classical ascription of actual values to these parameters. At first consideration this may appear churlish. There is an apparent difference between a sequence determined only by the free will of a Martian and a physical value. We ieave aside the question of whether this is an actual difference because this question misses the point. The point is to ask, "How can we assign meaning to statements concerning such quantities, in particular how should we understand quantification over such quantities?" Our refusal amounts to denying the coherence of any explanation based on the assumption that every sentence has a determinate truth value, either true or false. We refer to Dummett for elaboration of this point.

The possible results of experiments are concrete however. In general we may find by experiment that $a \in U$. These possible $U$ form a poset $\mathbb{P}$. The conceit which assumes that arbitrary refinement of our methods is in principle possible is represented by saying that the $V_{i} \leq U$ representing a certain degree of refinement cover U.

For example, measurement of a quantity classically represented by a real parameter could be represented by taking for $\mathbb{P}$ the poset of rational open intervals, with the stipulation that for each $\varepsilon>0$ an open interval $U$ is covered by the collection of all subintervais of length $\leq \varepsilon$, also that the collection of all proper subintervals of $U$ covers $U$.

In general then we consider a poset $\mathbb{P}$ of possible outcomes for an experiment. We ask, as a technical convenience, that if $p$ and $q$ represent a priori compatible results (i.e. if there is an $r$ with $r \leq p$ and $r \leq q$ ) then we can consider the outcome which consists just of getting these two results (i.e. we have an infinium $p \wedge q \in \mathbb{P}$ ). We also consider no information as a possible result (i.e. $\mathbb{P}$ has a 
top element T). The poset IP is equipped with a notion of covering family. We demand that this be

\author{
reflective \\ $\{p\}$ covers $p$ \\ stable \\ If $U$ covers $p$ and $q \leq p$ then $\{q \wedge w \mid w \in U\}$ covers $q$ \\ monotone \\ If $V \geq U$ covers $p$ then $V$ covers $p$.
}

The notion of a covering family is crucial to our explanation of meaning for incomplete objects. It formalises the sense in which they are potentially infinite. We avoid the metaphor of Wright [1981] which represents such a covering family as embodying the recognition that the state of information is capable of effective enlargement to one of type $a^{\star}\langle n\rangle$ because it seems to leave open to us the choice of not performing this enlargement. The idea we have is to introduce consideration of a particular type of incomplete object by specifying the type of data which generates it. This specification includes a notion of covering family. Differences over which is the proper collection of covering families do not affect the basic conception but merely lead to different types of data.

We are not as mathematicians or logicians interested in the result of a particular experiment. Rather, we are interested in those properties which would remain invariant no matter what the outcome or methodology of a particular experiment. It is not the result but the uses to which the result might be put in defining mathematical quantities which interest us. Were the temperature scale non-linear, or the time scale given by the unequal time of the sun, physics would be different (it was). But mathematics and logic should be immune to such vagaries.

Our solution is similar to that we employed in giving an objective view of open data. The possibility we envisage is that of a change of scale which in some sense refines our possibilities for measurement. The measurements of the old context should be meaningful in the new one but the new one may afford finer distinctions. To describe such a change of scale is to say which new observations $q \in \mathbb{Q}$ are to be viewed as refining an old observation $p \in \mathbb{P}$. We write this relation $q \leq f^{\star}(p)$ and demand that it be

monotone

$\frac{q^{\prime} \leq q \leq f^{*}(p) \quad p \leq p^{\prime}}{q^{\prime} \leq f^{*}\left(p^{\prime}\right)}$

multiplicative

$$
\frac{q \leq f^{*}(p) \quad q \leq f^{*}\left(p^{\prime}\right)}{q \leq f^{*}\left(p \wedge p^{\prime}\right)}
$$

continuous

$$
\frac{p_{i} \mid i \in I \text { covers } p \quad r \leq f^{\star}(p)}{\left\{q \mid q \leq f^{\star}\left(p_{i}\right) \text { some } i \in I\right\} \text { covers } r} \text {. }
$$

The motivation for the first two is clear. Continuity may be viewed as the requirement that a previous conviction that a certain family covers, cannot be overturned. The change of viewpoint induced by such a transformation $f$ is given by

$$
\frac{p ! \vdash \phi(\delta)}{q \Vdash \phi(\delta 1 f)} \frac{q \leq f^{*}(p)}{\text {. }}
$$

Mathematically, our notion of data gives a presentation of a zocaze. Change of scale is represented by a continuous function between locales. Abstractly we write such a change $f: Y \rightarrow X$.

We now consider a supplement to our notion of justification. Suppose, we consider, that consideration of a particular type of data would justify $\phi$, then $\phi$ is justified. This is the reflection on which our whole project is based: that we can justify talk of incomplete objects by reflecting on hypothetical indefinitely continued processes. 
We shall formulate this by saying that if $f: Y \rightarrow X$ represents the introduction of new distinctions independent of those represented by $X$ then

$$
\frac{Y ! \vdash \phi i f}{X \Vdash \phi}
$$

or that such an $f$ is a cover.

Our final problem of formalisation is to characterise the introduction of independent data. A simple example is, given $\mathbb{P}$ and $\mathbb{Q}$ with notions of covering, to consider $\mathbb{P} \times \mathbb{Q}$ the product poset with coverings

$$
\begin{aligned}
& \left\{\left\langle p_{i}, q>\right| i \in I\right\} \text { covers } p, q \text { when }\left\{p_{i} \mid i \in I\right\} \text { covers } p \\
& \left.\left\{\left\langle p, q_{j}>\right| i \in I\right\} \text { covers } p, q \text { when } q_{j} \mid i \in I\right\} \text { covers } q .
\end{aligned}
$$

The projection given by $\langle p, q\rangle \leq \pi^{*}\left(p^{\prime}\right)$ iff $p \leq p^{\prime}$ represents the introduction of data of type $\mathbb{Q}$ independently of the data $\mathbb{P}$ under consideration. We shall require that all such projections be covers.

In general there are two conditions we require to view a change of scale as the introduction of independent data. The first is obvious: no new covers should be introduced between existing observations.

$$
\frac{q_{i} \leq f^{\star}\left(r_{i}\right) \quad\left\{q_{i} \mid i \in I\right\} \text { covers each } q \leq f^{\star}(r)}{\left\{r_{j} \mid i \in I\right\} \text { covers } r} .
$$

The second is subtle: no new conditional relationships should be introduced between existing observations. We explain: if $w \in \mathbb{Q}$ is such that

$$
\frac{r \leq f^{\star}(p) \quad r \leq w}{r \leq f^{\star}(q)}
$$

(we view $w$ as establishing a conditional relationship between $f *(p)$ and $f *(q)$ ), we demand that $w \leq f *(s)$ for some $s \in \mathbb{I P}$ such that

$$
\frac{r \leq p-r \leq s}{r \leq q}
$$

(that the relationship be already established in $\mathbb{P}$ ). Technically, these requirements amount to demanding that the continuous map $f: Y \rightarrow X$ be a surjection and that it be open. The structure of data we have arrived at may be viewed as the category of locales equipped with the topology of covering by open maps.

Before turning to a formal examination of the interpretation of logic over this site, we sum up our intentions.

We introduce non-constructive objects by explaining the meanings of the connectives for statements concerning them. This is not a matter of characterising a domain of quantification. We have to explain the connectives anew in terms of the way such an object is given to us. Moreover, it is not sufficient to merely paraphrase the new quantifiers $\forall \alpha$ and $\exists \alpha$. Such a paraphrase entails a revision of the interpretations of $\rightarrow$ and $v$.

Our aim is to show that it is possible to derive rigorously properties of various domains of incomplete objects by giving a formal representation of the data which presents them as a site. We consider that the passage from an informal notion of data to the corresponding site is simple and natural. (Indeed, for us, to have a clear conception of a type of data is to be able to describe the corresponding site.) Once this passage is made, the derivation of properties (choice and continuity principles, for example) is a mathematical matter. Our hope in presenting these modeis is Leibnitzian: to eliminate further discussion of the justification of such principles by reducing the matter to calculation.

In our paper "Notions of Choice Sequence" [1982] we presented various notions of 
choice sequence, including ones satisfying the axioms of LS and CS, with the same purpose. Unfortunately, as the literature on choice sequences makes clear, clarity is in the eye of the beholder. Hence the present attempt at a more careful explanation of our informal notion of non-constructive object and its formalisation.

\section{CONTINUOUS TRUTH}

We start with a concrete presentation of the interpretation of higher-order logic in a Grothendieck topos. This material (\$\$1-3) is well-known to cognoscenti (tautologously), but is otherwise accessible only through a study of scattered references. We give some of these sources but make no systematic attempt at a complete list. Many important and historically significant contributions are not mentioned. Our account is fuller than is logically necessary for the sequel in order to point out some connections between different approaches. It is not, however, exhaustive.

\section{$\$ 1$ Frames and Locales}

1.1 Definition. A frome is a complete lattice with finite $\wedge$ distributive over arbitrary V. Frame morphisms, "and-or maps", are maps preserving these operations; $\mathrm{T}, \wedge, \mathrm{V}$.

1.2 Example. The lattice $O(X)$ of open subsets of a topological space is a frame. If $f: Y \rightarrow X$ is a continuous map then the inverse image $f^{\star}: O(X) \rightarrow O(Y)$ is an $\wedge, \bigvee$-map.

1.3 Definition. The category of Zocates or generalised spaces is the dual of the category of frames. We call the morphisms continuous maps $f: Y \rightarrow X$ and write $f^{\star}: O(X) \rightarrow O(Y)$ for the corresponding inverse image maps between the frames of opens of $X$ and $Y$ (as in the topological case).

Example 1.2 gives a functor $B:$ Top $\rightarrow$ Loc from topological spaces and continuous maps to locales.

1.4 Discrete spaces.

Spatialiy $P(A)$ corresponds to the discrete topology on $A$. An example is the onepoint space with $O(\mathbb{I})=P(\mathbb{I})$.

1.5 Definition. A topological space $X$ is sober iff $\operatorname{Top}[\mathbb{1}, X] \cong \operatorname{Loc}[\mathbb{1}, \beta X]$.

1.6 Lemma. On the fult subcategory of sober spaces $\beta$ is full and faithful.

We tacitly restrict our attention to sober spaces and henceforth omit mention of $B$. We view locales as generalised spaces. (The relationship between Loc and Top is better expressed in terms of the right adjoint, pt: Loc $\rightarrow$ Top, to $\beta_{\text {.) }}$

1.7 Subspaces. Quotient maps of frames induce congruences: if $f^{\star}: O(X) \rightarrow O(Y)$ is surjective Tet $p \approx q$ iff $f^{\star} p=f^{\star} q$. Each congruence class [p] has a canonical representative $j p=V\{q \mid p \approx q\}$. The maps $j: O(X) \rightarrow O(X)$ arising in this way are

monotone

idempotent

muztiplicative

$$
\begin{gathered}
p \leq j p \\
j^{2}=j \\
j(p \wedge q)=j p \wedge j q .
\end{gathered}
$$

Such maps are called nuctei. The quotient may be identified as the image (or fixed points) of $j$. The quotients of $O(X)$ are isomorphic (as posets) with the nuclei on $O(X)$. Spatially we view these quotients as giving rise to subspaces of $X$. 
1.8 Surjections. Dually, we view injective inverse image maps as giving rise to surjections of spaces.

1.9 Right adjoints. Each frame map $f^{*}$ has a right adjoint $f_{\star}$, direct image, given by

$$
f_{\star} p=V\left\{q \mid f^{\star} q \leq p\right\} \text {. }
$$

The map $q \mapsto p \wedge q$ has a right adjoint $r \mapsto p \rightarrow r$ defined by

$$
p \wedge q \leq r \quad \text { iff } q \leq p \rightarrow r
$$

where $p \rightarrow r=V\{q \mid p \wedge q \leq r\}$. Thus frames are complete Heyting algebras (but the morphisms are different).

1.10 Definition. A map of spaces $f: X+Y$ is open if the inverse image map $f^{\star}: O(Y) \rightarrow O(X)$ has a left adjoint $\exists_{f}: O(X) \rightarrow O(Y)$ commuting with $\wedge$ :

or, equivaiently, if $f^{\star}$ preserves $\rightarrow$.

$$
\exists_{f}(f *(y) \wedge x)=y \wedge \exists_{f}(x)
$$

1.11 Proposition. The category of locales is complete and cocomplete. Open surjections are stable (under pul1-back).

The theory of locales is developed extensively by Joyal and Tierney [1982].

Johnstone [1982] uses locales systematically and has a comprehensive bibliography.

$\$ 2$ Sites and Sheaves

2.1 Definitions. Let $\mathbb{C}$ be a small category. A cribze $K$ of $A \in|\mathbb{C}|$ is a subfunctor of the representable functor $A \in S^{\mathbb{C}^{\circ}}$ : that is, for each $B \in|\mathbb{C}|$ a set $K(B) \subseteq[B, A]$, stable under composition; for each $f \in K(B)$ and $g: C \rightarrow B$ in $C$, the composite $f \circ g \in K(C)$.

2.2 Lemma. The cribles of A form a frame, $P(A)$.

If $f: B \rightarrow A$ in $\mathbb{C}$ we have an inverse image map $f^{\star}: P(A) \rightarrow P(B)$ given by $f^{\star} K=\{g \mid f \circ g \in K\}$ for $K \in P(A)$. By abuse we write $f: B \rightarrow A$ for the corresponding continuous map. This map is open.

2.3 Definition. (Lawvere-Tierney) A Grothendieck topology $j$ on $\mathbb{C}$ is a family of nuclei $j_{A}: P(A) \rightarrow P(A)$, natural in $A$ : that is $f^{\star} \circ j_{A}=j_{B} \circ f^{\star}$ for $f: B \rightarrow A$.

2.4 Lemma. If $j$ is a Grothendieck topolngy on $\mathbb{C}$, the quotient frames $\Omega(A)$ have induced inverse image maps $f^{*}: \Omega(A) \rightarrow \Omega(B)$ and the corresponding map of locales, which we write $f: B^{j} \rightarrow A^{j}$, is open.

2.5 Definitions. A pretopology $J$ on $\mathbb{C}$ is a family $J(A) \subseteq P(A)$ for each $A \in \mathbb{C}$ which is

reflexive

$A \in J(A)$

multiplicative

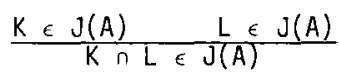

stable

$\frac{K \in J(A) \quad f: B \rightarrow A}{f^{\star} K \in J(B)}$.

(For example, let $K \in J(A)$ iff $j K=T$ ).

A crible $K \in P(A)$ is inductively closed for $J$ iff 


$$
f: B \rightarrow A \quad f^{*} K \in J(B) .
$$

As $A$ is closed and an intersection of closed cribles is closed, each crible $K \in P(A)$ has a closure $j_{A} K$. This gives a topology $j$ on $\mathbb{C}$. We say $K$ inductively covers $A$ iff $j K=A$, and write this $K \in \bar{J}(A)$.

2.6 Examples. (1) Let $O(X)$ be a frame viewed as a poset viewed as a category. Let $K \in J(A)$ iff $V K=A$. (Identifying $B \rightarrow A$ with its domain.) Then $\Omega(X) \cong O(X)$. (2) Let $\mathbb{C}$ be a small category of locales closed under finite limits and open inclusions. Let $K \in J(A)$ iff $K$ contains some family $\left\{f_{i}: B_{i} \rightarrow A \mid i \in I\right\}$ of open maps such that $V \exists_{f_{j}}\left(B_{j}\right)=A$. The crible generated by each open inclusion $U \subset A$ is closed for this topology. This assignment gives an $\wedge ; \vee$ map $r^{*}: O(A) \rightarrow \Omega(A)$, spatially a surjection $r: A^{j} \rightarrow A$. Each closed crible contains a largest open inclusion. This assignment gives an $\wedge \vee$ map $i^{\star}: \Omega(A) \rightarrow O(A)$, spatially we have an inclusion $i: A \Leftrightarrow A^{j}$. Furthermore, $r \circ i=i d_{A}$ and $r * i * \leq i d_{\Omega(A)}$ so $A$ is an adjoint retract of $A^{j}$ (Fourman [1982]).

2.7 Definition. A presheaf on $\mathbb{C}$ is a functor $X: \mathbb{C}^{O P} \rightarrow$ Sets. If $f: B \rightarrow A \in \mathbb{C}$ and a $\epsilon X(A)$ we use the notation alf, "a restricted along $f$ ", for $X(f)(a) \in X(B)$. Note that $\mathrm{a} \mid \mathrm{f} 1 \mathrm{~g}=\mathrm{a} 1 \mathrm{f} \circ \mathrm{g}$ and $\mathrm{a} \mid \mathrm{id}=\mathrm{a}$. The appropriate morphisms between presheaves $F: Y \rightarrow X$ are natural transformations, maps $F_{A}: Y(A)+X(A)$ which commute with restrictions $F_{B}(a \mid f)=\left(F_{A} a\right) 1 f$.

2.8 Examples. (1) The representable functor $[-, A]$, (or by abuse, justified by Yoneda's lemma, A) is a presheaf. Restrictions are by composition $g 1 \mathrm{f}=g \circ f$. Yoneda's lemma tells us that for any other presheaf, $X$, we have $X(A) \cong[A, X]$. In particular the embedding $\mathbb{C} \rightarrow S^{\mathbb{O P}}$ is full and faithfu?. Each crible $K \in P(A)$ is a subpresheaf $K \rightarrow A$.

(2) $P$ and $s$ are presheaves with restrictions given by inverse images $k 1 f=f *(K)$.

2.9 Definition. A presheaf $X$ on $\mathbb{C}$ is a sheaf for the (pre) topology $J$ if whenever $K \in J(A)$, each natural transformation $x: K \rightarrow X$ has a unique extension along $K>A$. Equivalently, if $K \in J(A)$ and we have a family $x_{f} \in X(B)$ for $f: B \rightarrow A \in K$ such that $x_{g f}=x_{f} \mid g$ for each $g: C \rightarrow B$, there is a unique $X \in X(A)$ such that $x_{f}=x 1 f$ for each $f \in K$. The catecorv of sheaves and natural transformations is a Grothendieck topos.

There is, as yet, no staisfactory introductory text on topos theory. The basic references are SGA4, Wraith [1975], Johnstone [1977], Freyd [1972].

\section{$\$ 3$ Forcing over a site}

Here we describe Joyal's presentation of interpretations in topoi in terms of a notion of forcing. Let $\mathbb{C}$ and a (pre) topology $J$ be fixed. The basic structures we consider are diagrams of preshecves on $\mathbb{C}$. Each presheaf $A$ interprets a type or sort of variable. A morphism $f: A_{1} \times \ldots \times A_{n} \rightarrow B$ interprets an n-ary operation. A subobject $R \nrightarrow A_{1} \times \ldots \times A_{n}$ interprets an n-ary relation.

3.1 Definitions. Let $L$ be a first-order language (possibly many-sorted) with equality. An interpretation of $L$ is given by assigning to each sort $A$ of $L$ a presheaf $A$, to each operation $F$ from $A_{1}, \ldots, A_{n}$ to $B$ a natural transformation $F: A_{1} \times \ldots \times A_{n} \rightarrow B$, and to each relation $R$ on $A_{1} \times \ldots \times A_{n}$ a subfunctor $R>A_{1} \times \ldots \times A_{n}$. Given such an interpretation, for $U \in|\mathbb{C}|$ we let $L_{U}$ be the 
expansion of $L$ ebtained by adding constants of the appropriate sorts for the elements of $A(U)$. If $f: V \rightarrow U$ then for any term $T$ or formula $\phi$ of $L_{U}$ we obtain a term $\tau \uparrow f$ or formula $\phi 1 f$ of $L_{V}$ by restricting any new constants which occur. Now for $U \in|\mathbb{C}|$ we define for each closed term $\tau$ of sort $A$ of $L_{U}$ an interpretation $\llbracket \tau \|_{U} \in A(U)$ by induction:

$$
\begin{gathered}
\|c\|_{U}=c \quad \text { for } c \in A(U) \\
\| F\left(\tau_{1}, \ldots, \tau_{n}\right) \rrbracket_{U}=F\left(\| \tau_{1} \rrbracket, \ldots,\left[\tau_{n} \rrbracket\right) .\right.
\end{gathered}
$$

Note that $\llbracket \tau \| f \rrbracket_{V}=\llbracket \tau \rrbracket_{U} 1 f$. Now we define inductively the relation, $U$ forces $\phi$, $U \Vdash \phi$

for $\phi$ a sentence of $L_{U}$.

INDUCTIVE DEFINITION OF FORCING

$$
\frac{V_{f} \| \phi 1 f \text { al1 } f: V_{f} \rightarrow K}{U \Vdash \phi} \quad K \in J(U)
$$

$(\wedge)^{+}$

$$
\frac{U \Vdash \phi}{U \Vdash \phi \wedge \psi}
$$

$(v)^{+}$

$$
\frac{U \Vdash \phi}{U \Vdash \phi \vee \psi} \quad \frac{U \Vdash \psi}{U \Vdash \phi \vee \psi}
$$

$(3)^{+}$

$$
\frac{U \Vdash[c / x]}{U \Vdash \exists x . \phi}
$$

$(\rightarrow)^{+}$

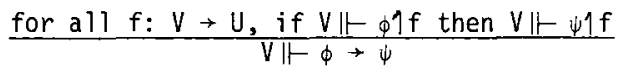

$(\forall)^{+}$

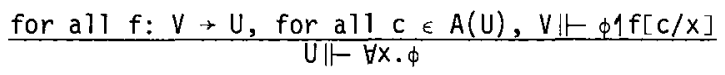

$(=)^{+}$

$$
\frac{\llbracket \tau_{1} \rrbracket=\llbracket \tau_{2} \rrbracket}{U \Vdash-\tau_{1}=\tau_{2}}
$$

(Atomic) $^{+}$

$$
\frac{\left\langle\| \tau_{1} \mathbb{l}, \ldots, \mathbb{\|} \tau_{n} \rrbracket>\epsilon R\right.}{U \Vdash R\left(\tau_{1}, \ldots, \tau_{n}\right)}
$$

We now give some "derived rules" for forcing:

3.2 Lemma. Basic properties of forcing

$$
\frac{U \Vdash \phi \quad f: V \rightarrow U}{V \|-\phi I f}
$$


$(\Lambda)^{-}$

$\frac{U \Vdash \phi \wedge \psi}{U \Vdash \phi} \hat{U \Vdash \psi}$

$(v)^{-}$

$U \Vdash \phi \vee \psi$

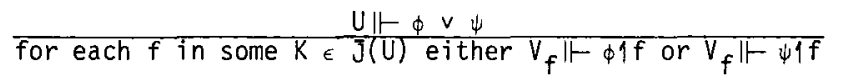

$(\exists)^{-}$

for each $f$ in some $K \in J(U)$ we have $V_{f} \mathbb{H} \phi[c / x]$ for some $c \in A\left(V_{f}\right)$

$(\rightarrow)^{-}$

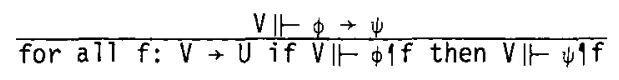

$(\forall)^{-}$

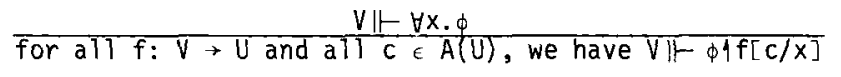

$(=)^{-}$

$$
\begin{aligned}
& U \| \tau_{1}=\tau_{2} \\
& \text { for each } f \text { in some } K \in J(U) \text { we have }\left\|\tau_{1} \rrbracket / f=\mathbb{\|} \tau_{2}\right\| 1 f
\end{aligned}
$$

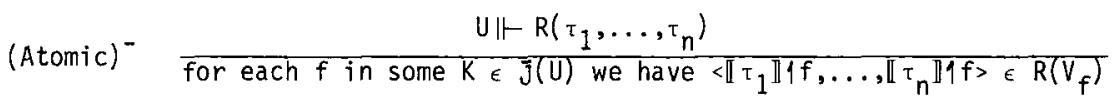

Our presentation here is non-standard in that the definition of forcing is usually given by stipulating both positive and negative rules for each connective, (I) and $(P)$ are then derived. The resulting relation is the same.

3.3 Definition. A sequent $\Gamma \Rightarrow \phi$ is valid (written $\Gamma \neq F_{\phi}$ ) in the given interpretation iff

$$
\frac{U \Vdash H[\xi(x) / x] \text { all } \psi \in \Gamma}{U \| \phi[\xi(x) / x]}
$$

where $\xi$ is an interpreation of the variables of $L$ by elements of the appropriate $A(U)$.

3.4 Proposition. If each sort is interpreted by an inhabited presheaf (each A(U) is inhabited) then the axioms and rules of Heyting's propositional calculus are valid for $\equiv$.

(Adaptations for domains which are not inhabited are discussed in Fourman [1977], Scott [1978], Joyal \& Boileau [1981], Makkai \& Reyes [1977].)

3.5 Definitions. A presheaf $A$ is separated iff

$$
\frac{U \|-a=b}{a=b} \quad \text { for } a, b \in A(U) \text {. }
$$

A subobject $R \rightarrow A$ is closed iff

$$
\frac{U \Vdash R(a)}{a \in R(U)} \quad \text { for } a \in A(U) \text {. }
$$

A higher-order type-theory is merely a many-sorted first-order theory with some structure on the collection of sorts and certain distinguished operations and relations. One of the insights due to Lawvere and Tierney is that topoi have such higher-order structure. We consider languages where for any two sorts $A$ and $B$ we can form the product $A \times B$ with appropriate pairing and projection operations, the function space $B^{A}$ with an evaluation operation $-(-)$, and also the power type 
$P(A)$ with a membership relation $\epsilon$. An interpretation is standard if all this structure is interpreted by the corresponding structure on $\operatorname{Sh}(\mathbb{C})$.

3.6 Proposition. In any standard interpretation the following schemata, which combine comprehension and extensionality, are valid.

$$
\begin{gathered}
x \in A \quad \exists ! y \in B . \phi(x, y) \leftrightarrow \exists ! f \in B^{A} \forall x \in A . \phi(x, f(x)) \\
\exists ! z \in P(A) \forall x \in A \quad(x \in Z \leftrightarrow \phi(x)) .
\end{gathered}
$$

Thus power-types and function spaces behave as they should. The categorical characterisation of this higher-order structure in terms of adjoints is very simple, products are categorical products,

$$
\frac{C \rightarrow B^{A}}{A \times C \rightarrow B} \quad \frac{C \rightarrow P(A)}{\operatorname{Sub}(A \times C)}
$$

We shall not describe this structure in general here. We shall be dealing primarily with sorts interpreted by representables. These are particularly simple to deal with because they have generic ezements. A well-known consequence of this is the Yoneda Lemma:

$$
F(U) \cong[U, F] \text { for } F \in\left|S^{\mathbb{C}}\right| \text { and } U \in|\mathbb{C}| \text {. }
$$

We use this to calculate some examples of the higher-order structure. For this exercise, we suppose that $\mathbb{\complement}$ has finite products and that each representable functor is a sheaf.

3.7 Lemma. If $F$ is a sheaf and $U, V$ are representable

(1) $F^{U}(U) \cong F(U \times C)$

with evaluation for $u: V \rightarrow U$ and $\xi \in F(U \times V)$ given by $\xi(u)=\xi \mid\langle u, V\rangle$.

(2) $(P U)(V)=\Omega(U \times V)$ with $U \Vdash a \in R$ iff $R \mid<a, V>=V$.

Proof. $F^{U}(V) \cong\left[V, F^{U}\right] \cong[U \times V, F] \cong F(U \times V)$

$$
(P U)(V) \cong[V, P U] \cong \operatorname{Sub}(U \times V)=\Omega(U \times V) \text {. }
$$

A logical counterpart to Yoneda's lemma is the following.

3.8 Lemma. Generic elements for representabies.

If $U$ is representable then $V \| \forall x \in U . \phi$ iff $V \times U \| \phi 1 \pi_{1}\left[\pi_{2} / x\right]$.

Proof. In one direction this is immediate from $(\forall)^{-}$. In the other, suppose f: $W \rightarrow V$ with $a: W \rightarrow U \in U(W)$ then $\langle f, a\rangle: W \rightarrow V \times U$ and, by persistence, if $V \times U \| \phi \pi_{1}\left[\pi_{2} / x\right]$ then $W \|-\phi 1 f[a / x]$. So by $(\forall)^{+}$we have $V \| \forall x . \phi$.

We give an example of the use of generic elements in the simple case of a category of presheaves.

3.9 Proposition. Choice holds for representables in categories of presheaves. Proof. Let $U$ be a representable and suppose

$$
V \| \forall x \in U . \exists y \in F . \phi(x, y)
$$

then

$$
\begin{gathered}
U \times V \|-\exists y \in F . \phi 1 \pi_{2}\left(\pi_{1}, y\right) \\
U \times V \|-\phi 1 \pi_{2}\left(\pi_{1}, \xi\right) \text { for some } \xi \in F(U \times V)
\end{gathered}
$$


regarding $\xi$ as an element of $F^{U}(V)$ this gives

$$
U \times V \Vdash \phi 1 \pi_{2}\left(\pi_{1},\left(\xi \hat{q}_{2}\right)\left(\pi_{1}\right)\right) \text {. }
$$

Since $\xi 1<\pi_{1}, \pi \pi_{2}=\xi$. Thus

$$
V: \forall x \in U . \Phi(x, \xi(x)),
$$

and so

$$
V \| \exists f . \forall x . \phi(x, f(x)) .
$$

From a category-theoretic viewpoint this result is well-known in the form, "Representables are internally projective".

\section{§4 POINTS, LOCAL CHOICE, CONTINUITY}

Now we let $\mathbb{C}$ be a category of locales closed under finite limits and open inclusions, equipped with the open cover topology, J. We write $\mathbb{E}$ for the topos $\operatorname{Sh}(\mathbb{C}, J)$.

For each locale $X$ we define an internal locale $X$ by

$$
o(X)(u) \cong o\left(X \times u^{j}\right) \text {. }
$$

This is generated internally by the basis given by

$$
B(W)(U) \cong O(X \times U)
$$

or even by the constant basis

$$
B_{0}(X)(U)=O(X) \text {, }
$$

with the inclusions $B_{0}(\mathbb{X}) c_{\rightarrow} B(\mathbb{X}) \hookrightarrow O(\mathbb{X})$ induced by the projections $X \times U^{j} \rightarrow X \times U \rightarrow X . \quad$ (In the terminology of Joyal \& Tierney $X=P^{\star}(X)$.)

The internal space of points of $W$ is given by

$$
(p t X)(U) \cong C\left[U^{j}, X\right] \text {. }
$$

This is the space of $\mathbb{E}$-valued models of $X$. For $\alpha: U^{j} \rightarrow X$ and $W \in O\left(X \times U^{j}\right)$

$$
U \|-\alpha \in W \text { iff }\left\langle\alpha, U^{j^{-1}}(W)=T\right. \text {. }
$$

For $\alpha: U^{j} \rightarrow X$ and $V \in O(X \times U)$

$$
\begin{aligned}
U \| \alpha \in V & \text { iff }\left[(X \times r)_{0}<\alpha, U^{j}>\right]^{-1} V=T \\
\text { iff } \quad[X \times \alpha & 0 i]^{-1} V=T
\end{aligned}
$$

since $j^{\star}: O\left(U^{j}\right) \rightarrow O(U)$ reflects $T$.

4.1 Proposition. For any $X \in|\mathbb{C}|$, the internal locale $\mathbb{X}$ has enough points.

Proof. We must show for $W \in O(X)$ that

$$
\underline{U \| K} \text { covers pt } W \quad U \mathbb{U} \text { U is an inductive crible. }
$$

We assume the hypotheses, and let

$$
\mathbb{K}=\left\{W_{i} \times U_{i} \mid U_{i} \Vdash W_{i} \in K\left\{U_{i}\right\}\right.
$$

Clearly $\mathbb{K}$ is a doubly inductive crible of $O(W) \times O(U)$, that is an open of $W \times U$. We show that IK covers $W \times U$ that is that $W \times U \in K$, which is evidently sufficient since then $U \| W \in K$.

By persistence

$$
W \times U \Vdash K 1 \pi_{2} \text { covers } \pi_{1}
$$


that is

$$
W \times U \Vdash \exists V \in K\left\{\pi_{2} \cdot \pi_{1} \in V\right.
$$

So

$$
\mathbb{I K}^{*}=\left\{W_{i} \times U_{i} \mid \text { for some } V_{i} \in O(W) \text { we have } w_{i} \times U_{i} \Vdash V_{i} \in K\left\{\pi_{2} \wedge \pi_{1} \in V_{i}\right\}\right.
$$

covers $W \times U$. [Because, if $p: X \rightarrow Y$ is an open surjection and $X \| V \in K|p \wedge \theta| p \in V$ then $Y \mathbb{F} V \in K \wedge \theta \in V$ : the basic opens of $\mathbb{X}$ are constant and thus descend open surjections.] But now we claim $\mathbb{I K}^{*} \subseteq \mathbb{I}$ because, by definition

$$
w_{i} \times u_{i} \Vdash \pi_{1} \in V_{i} \text { iff } w_{i} \subseteq V_{i}
$$

and, as projections are covers,

$$
W_{i} \times U_{i} \Vdash V_{i} \in K \|_{2} \text { iff } U_{i} \Vdash V_{i} \in K U_{i} .
$$

Special cases of this are worthy of mention. When $X$ is Baire space $N^{N}$, Cantor space $2^{N}$, Dedekind reals $R$, to say that $X$ has enough points is the internal statement of Bar induction, Fan theorem, Heine Borel theorem (respectively). For these cases it is sufficeint to take the topology on $C$ generated by covering families of open inclusions: since each of these spaces $X$ has $\alpha$ point the proections $X \times U \rightarrow X$ are covers for this topology. We call this topology the open inclusion topology.

We introduce some more general spaces. Let $f: X \rightarrow U$ in Loc. We consider the internal locale $X / f$ defined at $U$ by the basis $O(V)$ with all its standard covers. More properly for $g: W \rightarrow U$ we define

$$
B(x / f) \mid g \cong O\left(g^{\star} x\right)
$$

given by pulling back $f$ along $g$. Any commuting triangle

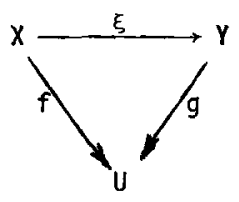

induces an internal map of locales

$$
\xi: X / f \rightarrow Y / h
$$

defined at $U$. Given by $\xi^{-1}$ on basis elements, this clearly takes basic covers to covers. Furthermore, if $\xi: X \rightarrow Y$ is open (and surjective) then $\xi: X / f \rightarrow Y / h$ is open, since it suffices to define $\exists_{\xi}$ commuting with $\wedge$ on basis elements, (and surjective since if $\xi: X \rightarrow Y$ is an open surjection then so are all its pullbacks, so internally $\xi^{-1}$ reflects basic open covers). These spaces include the spaces $\mathbb{W}$ we introduced earlier as

$$
U \Vdash \mathbb{W} \cong(\mathrm{X} \times \mathrm{U}) / \pi \text {. }
$$

We now specialise to the case where the objects of $C$ are $T_{1}$. Then $U \hookrightarrow U^{j}$ induces an isomorphism

$$
(p+\not)(U) \cong c\left[U^{j}, X\right] \cong c[U, X]
$$

so that $X$ represents the functor pt $X X$. This happens in particular for the spaces $N, N^{N}, 2^{N}, R$ and their basic opens (see Fourman [1983].) Furthermore, any element of $p t(X / f)$ defined at $U$ induces a commuting triangle 


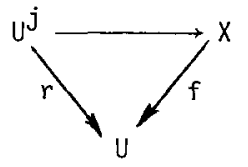

$$
\begin{aligned}
& \text { which for } T_{1} \text { spaces } \\
& x \text { corresponds to a } \\
& \text { section of } g
\end{aligned}
$$

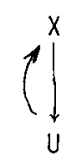

So we have a presentation

$$
\operatorname{pt}(X / f) \mid g \quad \begin{aligned}
& \text { correspond to } \\
& \text { commuting triangles }
\end{aligned}
$$

with restriction given by composition.

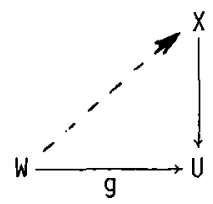

We extend our earlier lemma on generic elements:

4.2 Lemma. If objects of $\mathbb{C}$ are $T_{1}$ then

$$
U \Vdash \forall x \in \operatorname{pt}(x / f) \text {. } \phi
$$

iff

$$
x \Vdash(\phi \mid f)(\text { id) } .
$$

of course these generalised representables can be defined internally in any Grothendieck topos and this result holds.

4.3 Proposition. If the objects of $\mathbb{C}$ are $T_{1}$ then for any $X \in|\mathbb{C}|$ and any $A \epsilon \operatorname{Sh}(\mathbb{C})$

$\vDash \forall x \in \operatorname{pt}(\mathbb{X}) . \exists a \in A \cdot \phi(x, a)$

$\rightarrow \exists$ open cover $p: Z \rightarrow \mathbb{W}$ and a function $f: p t Z \rightarrow A$ such that

$\forall x \in A \cdot \forall z \in$ pt $Z[p z=x \rightarrow \phi(x, f(z))]$.

Proof. As pt $\mathbb{W}$ is representable,

$$
U \Vdash \forall x \exists a \phi(x, a)
$$

iff

iff for some open cover $p: Z \rightarrow X \times U$

$$
X \times U \Vdash \exists a \phi 1 \pi_{2}\left(\pi_{1}, a\right)
$$

$$
Z \Vdash \phi 1 \pi_{2}{ }^{\circ p}\left(\pi_{1}{ }^{\circ p, \xi)} \text { for some } \xi \in A(Z)\right.
$$

iff

$$
U \| \forall Z \in Z \phi(p(z), \xi(z)) \text {. }
$$

We do not know under what conditions $\xi$ descends to give a function defined on a cover by open sets. We can ensure this by considering the open inclusion topology on $\mathbb{C}$ in which case we obtain

$$
\begin{aligned}
& F \forall x \in \text { pt } X . \exists a \in A \cdot \phi(x, a) \\
& +\exists \text { open cover } U_{i} \in O(x) \text { and functions } f_{i}: U_{i} \rightarrow A \text { such that } \\
& \forall x \in U_{i} \cdot \phi\left(x, f_{i}(x)\right) .
\end{aligned}
$$

We now consider continuity.

4.4. Proposition. If $X, Y$ are $T_{1}$ then $\vDash \forall f: p t W \rightarrow p t W, f$ is continuous. 
Proof. If $U \Vdash f: p t W \rightarrow p t \mathbb{W}$ then $f$ is represented by $\xi: X \times U \rightarrow Y$ in $\mathbb{C}$. For $\overline{V \in O}(Y)$ a basic open of $W, W: W \rightarrow U$ and $X: W \rightarrow X$ we have

$$
W \Vdash(\xi \mid w)(x) \in V
$$

iff

$$
[\xi \circ<x, W>]^{-1}(V)=W
$$

iff

$$
\langle x, w\rangle^{-1} \xi^{-1}(v)=w
$$

iff

$$
w \Vdash x \in\left[\xi^{-1}(V) \mid w\right]
$$

regarding $\xi^{-1} V \in O(X \times U)$ as an open of $\mathbb{W}$ defined at $U$.

Thus $U \| \xi^{-1}(v)$ is open.

\section{$\$ 5 \quad$ Iteration}

We return for a while to consideration of a general Grothendieck topos $\mathbb{E}=\operatorname{Sh}(\mathbb{C}, J)$. We consider the internal category $\mathbb{C}$ in $\mathbb{E}$ given by

$$
\mathbb{L}(\mathrm{U}) \cong \mathbb{C} / \mathrm{U}
$$

with restrictions given by pulling back.

[For those who worry about coherence (one should worry), we remark that a concrete category in $\mathbb{E}$ with an equivalent category of sections over $U$ is given by considering $V / f$ to be represented as the element $S$ of $(P V)(U)$ determined by

$$
w \Vdash v \in S l g \text { iff } f \circ v=g \text {. }
$$

So $\mathbb{d}$ is an internal small full subcategory of $\mathbb{I}$ whose objects are subfunctors of representables.]

We give $\underset{\sim}{C}$ a topology by letting

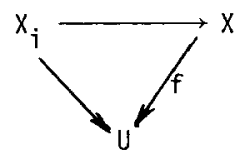

cover $x / f$ in $\mathbb{d}$ if $X_{i} \rightarrow x$ cover $x$ in $\mathbb{w}$.

Now for $A \in|\mathbb{E}|$ we define $\underset{\sim}{A} \in S h_{\mathbb{E}}(C, J)$ by

$$
U \| A(X / f) \cong A(X)
$$

with restrictions for $g: V \rightarrow U$ given by restriction along $f^{\star} g$

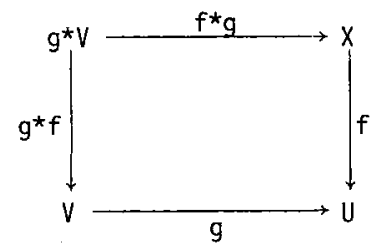

and for $\xi: Y / h \rightarrow X / g$ in $\mathbb{C} / U$, by restriction along $\xi$

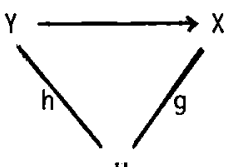

U

Any morphism $A \rightarrow B$ in $\mathbb{E}$ induces $\underset{\sim}{A} \rightarrow \underset{\sim}{B}$ in $\operatorname{Sh}_{\mathbb{E}}(\underset{\sim}{\mathbb{C}}, J)$. 
For those who prefer global descriptions, we associate to $A \in|\mathbb{E}|$ (pseudo) functors

$$
\mathbb{C} / U \rightarrow \mathbb{E} / U
$$

natural in $U$ (i.e. commuting with $g^{*}$ for $g: V \rightarrow U$ ) as follows:

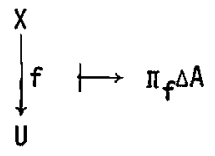

where

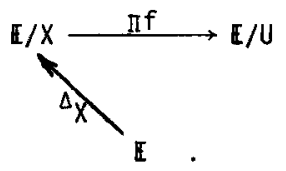

For $Y \longrightarrow \underline{\xi} \longrightarrow X$ we have $\pi_{h} \cong \pi_{f} \Pi_{\xi}$ whence $\Pi_{h} \xi^{*} \Rightarrow \pi_{f}\left(\right.$ as $\left.\xi^{*}+\pi_{\xi}\right)$

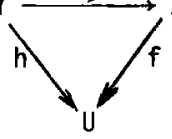

and $\Pi_{h} \Delta_{Y} \Rightarrow \pi_{f^{\Delta}} \Delta_{X} \quad\left(\right.$ as $\left.\xi^{\star} \Delta_{X} \cong \Delta_{Y}\right)$.

This gives the required arrow $\pi_{h}{ }^{\Delta} Y A \rightarrow \pi_{f}{ }^{\Delta} X^{A}$. What we obtain is an (internal) functor

$$
E \rightarrow \mathbb{E}^{C^{o p}} \text {. }
$$

We shall show that this preserves first order logic.

Here we work concretely for the sake of computations. A simple but more abstract treatment wil1 appear in Fourman and Kelly [1983]. We now consider a first-order language $L$ with sorts for the objects of $\mathbb{E}$ and operations symbols for its morphisms. In fact to avoid size problems, we consider an arbitrary small fragment of such a language. We may consider $L$ also as a language in $\mathbb{E}$ as a constant object (via $\Delta$ ).

Working in $\mathbf{E}$ we consider the interpretation of $L$ given by interpreting the sort $A$ by $A$ and each operation $f: A \rightarrow B$ by the corresponding morphism $A \rightarrow B$.

5.1 Lemma. For $f: X \rightarrow U$ and $g: X \rightarrow V$

$$
U \Vdash X / f \mathbb{R} \quad \text { iff } \quad V \Vdash X / g \Vdash \phi
$$

Proof. By induction, it suffices to show that if $U \Vdash \mathbb{R} / f \|{ }^{*} \phi$ is defined to mean

$$
V \| X / g \Vdash \phi \text { for all } g: X \rightarrow V
$$

then $\|^{*}$ is closed under the rules of . As no rule decreases the complexity of $\phi$ we may assume that the result holds for subformulae of $\phi$.

Oniy $(\rightarrow)^{+}$and $(\forall)^{+}$present any difficulties. We consider $(\rightarrow)^{+}$, and suppose the result for $\phi$ and $\psi$.

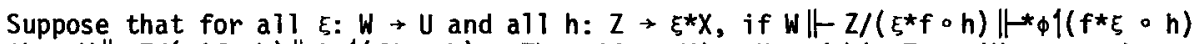
then $W \| Z /\left(\xi^{*} f h\right) H^{*} w^{\prime}\left(f^{\star} \xi \circ h\right)$. Then if $\eta: W^{\prime} \rightarrow V$ and $h^{\prime}: Z \rightarrow \eta^{\star} x$ are such that $W^{\prime} \mid-Z^{\prime} /\left(n^{*} g \circ h^{\prime}\right) \| \phi^{\prime}\left(g^{*} n^{\circ} \circ h^{\prime}\right)$ then by induction hypothes is 


$$
U \Vdash Z^{\prime}\left(f \circ g^{*} \eta \circ h^{\prime}\right) H^{*} \phi 1\left(g^{*} \eta \circ h^{\prime}\right)
$$

whence (letting $\xi=i d$ and $h=g^{\star} n \circ h^{\prime}$ ) we have

$$
u \Vdash z^{\prime} /\left(f \circ g^{\star} n \circ h^{\prime}\right) \Vdash \psi^{*} 1\left(g^{\star} \eta \circ h^{\prime}\right)
$$

in particular $W^{\prime} \Vdash Z^{\prime}\left(n^{*} g \circ h^{\prime}\right) \Vdash \psi^{\prime}\left(g^{*} \eta^{\circ} \circ h^{\prime}\right)$. So $V \Vdash x / g \Vdash \phi \rightarrow \psi$. The proof for $\forall^{+}$is similar.

\subsection{Theorem. For $\phi$ a formula of $L$ with appropriate parameters}

$$
U \Vdash \| x / f \Vdash \phi " \text { iff } X \Vdash \phi \text {. }
$$

Proof. Firstly, this is well formed: Parameters for $\phi$ at $X / f$ are elements of $\bar{A}(X / f)$ which are given as elements of $A(X)$ and are thus parameters for $\phi$ at $X$.

We proceed by induction. That is, we show that if we define $H^{*}$ internally by

$$
U \| x / f H^{*} \phi \quad \text { iff } x \| \phi
$$

then $\|^{*}$ is closed under the defining clauses of $\|_{-}$internally, (whence

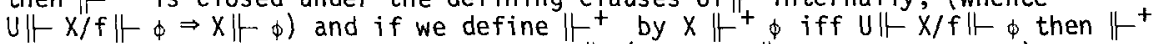
is closed under the defining clauses of $\Vdash$ (whence $x \| \phi \Rightarrow U \Vdash X / f \Vdash \phi)$.

As the operations $\underset{A}{A} \rightarrow$ are just those inherited from $\mathbb{E}$, terms are interpreted alike in both contêxts. Thus if $\llbracket \tau \rrbracket=\llbracket \sigma \rrbracket$ then $U \| \mathbb{V} \llbracket \rrbracket=\llbracket \sigma \rrbracket$, so $\mathbb{H}^{+}$is closed under $(=)^{+}$and if

so $\mathbb{F}^{*}$ is closed under $(=)^{+}$.

$$
U \Vdash \| \tau \rrbracket=\llbracket \sigma \rrbracket \text { then } U \Vdash \tau=\sigma,
$$

That $\mathbb{1}^{+}$and $\mathbb{H}^{*}$ are closed under $(\Lambda)^{+},(v)^{+},(\exists)^{+}$is trivial. For 1 , suppose $x_{i} \Vdash^{+}{ }_{\phi 1 f_{j}}$ for $f_{i}: x_{i} \rightarrow X$ in some cover of $x$ then $x \Vdash x_{j} / f_{j} \Vdash \phi 1 f_{i}$ and by $I$ internally $x \Vdash x / i d \Vdash \phi_{\phi}$. In the contrary direction, suppose $U \Vdash X_{i} / g \circ f_{j} \mathbb{H}^{*}{ }^{*} \phi f_{i}$ for some cover of $x$ as above. Then $x_{i} \Vdash|\phi| f_{i}$ so $x \Vdash \phi$ that is $U \Vdash X / g \Vdash \phi$. For $(\rightarrow)^{+}$, first suppose that for all $f: V \rightarrow U$ if $V \mathbb{H}^{+}{ }_{\phi} \mid f$ then $V \mathbb{H}^{+}{ }^{+} \phi \mid f$. Then we claim $U\|U / i d\| \phi \rightarrow \psi$, because for all $g: W \rightarrow U$ and all $h: V \rightarrow W$, if $W\|V / h\|-\phi 1 g \circ h$, then $V \mathbb{H}^{+} \psi 1 \mathrm{~g} \circ \mathrm{h}$ so $V \mathbb{H}^{+} \psi^{+} \mathrm{g} \circ \mathrm{h}$, that is $w\|v / h\| \psi 1 \mathrm{~g} \circ \mathrm{h}$. Conversely, if for all $g: W \rightarrow U$ and all $h: Z \rightarrow g^{\star} X$, where $f: X \rightarrow U$, if $W \Vdash Z / g^{\star} f \circ h \| \vdash^{*} \psi / f^{*} g \circ h$, then $x \Vdash \phi \rightarrow \psi$, because for $h: Z \rightarrow x$ if $Z$ H $\phi$ ih then

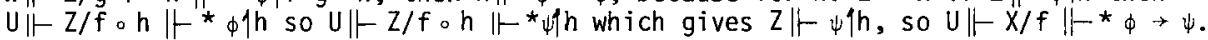
The proof for $\forall^{+}$is similar.

We view this thorem as asserting that in the topos $\mathbb{E}$ the naive notion of truth given by the equivalence thesis is consonant with the theory of meaning given by the notion of forcing over the site $\mathbb{C}$. Of course this may seem vacuous as it appears that $\mathbb{C}$ is manufactured with $\tilde{\text { this }}$ result in mind. However, in the case of primary interest for this paper, the results of $\$ 4$ allow us to regard $\mathbb{a}$ internally as a full subcategory of $\operatorname{Loc}(\mathbb{E})$ equipped with the open cover topology. 'In fact, if $\mathbb{G}$ is the category of separable locales, we may identify $\mathbb{d}$ as a category of separable locales in $\mathbb{E}$. We shall deal with this, among otherr things, in a sequel to this paper.

Given $f: X \rightarrow U$ we may view an element a of $A(X)$ as a function:

$$
U \| \mathrm{a}: X / f \rightarrow \underset{\sim}{A} \text {. }
$$

This allows us to rephrase our theorem.

$$
5.3 \text { Corollary. } U \Vdash X / f \mid F \phi(\bar{a}) \text { iff } U \Vdash \forall t \in X / f_{\phi}(\bar{a}(t)) \text {. }
$$

We view this as a general form of the elimination theorem (cf. Troelstra [1977] 
pp.33,79). The appropriate theory of continuous truth CT has an axiom for each clause in the definition of $X / f \| \phi(\bar{a})$. For example, the clause for $\exists$ gives the axiom of local choice $\forall t \in X \exists y \phi(\bar{a}(t), y)$ iff $\exists$ open cover $p: Z \rightarrow X$ and continuous $f: Z \rightarrow Y$ such that $\forall z_{\phi}(\bar{a}(p(z)), f(z))$. The translation $\tau \phi$ of a formula $\phi$ without free lawless variables is given by $\tau \phi \equiv$ def $\Vdash-\phi$.

\section{CODA}

A general notion of non-coristructive object is given by interpretations in Grothendieck topoi. The process of iteration described in $\$ 5$ shows how we may view (internal) truth in this interpretation as given by a non-standard theory of meaning. The clauses defining this give axioms for the corresponding theory of continuous truth CT and an "elimination" translation. By construction, CT $\vdash \phi \leftrightarrow \tau \phi$ and for formulae in the lawlike part of the language $\tau \phi \equiv \phi$. The proof theoretic content of the elimination;

$$
\text { CT } \vdash \text { iff ID } \vdash \tau \phi \text {, }
$$

requires formalisation of our treatment in an appropriate theory ID of inductive definitions. We do not undertake this here.

A final example of an unfinished object is this paper. Some of the results, in particular continuity principles in sheaves over sites, go back to 1978 and were much influenced by discussions with Scott and Hyland. Some results are still being refined. Other persistent influences have been those of Joyal and Lawvere on the one hand and of Kreisel, Troelstra and Dummett on the other. This research has been supported at various times by the N.S.F. (U.S.A.), the S.R.C. (U.K.), the Z.W.0. (Netherlands), and the A.R.G.S. (Australia), and made easier by the hospitality of many people notably Christine Fox, Irene Scott, Karen Green, and Imogen KelTy. I am grateful.

\section{REFERENCES}

Artin, M., Grothendieck, A., Verdier, J.L., Théorie des Topos et Cohomologie, Etale des Schémas (SGA4), (Lecture Notes in Math. 269, 270, Springer-Verlag, Berlin, 1972).

Beth, E.W., Semantical Considerations on Intuitionistic Logic, Indag. Math., $9(1947)$, p.572-7.

Boileau, André \& Joya1, André, La logique des topos, J.S.L. 46(1981), p.6-16.

Brouwer, L.E.J., Cambridge Lectures on Intuitionism, D. van Dalen, ed. (Cambridge University Press, 1981).

Dummett, Michael, Elements of Intuitionism, (Oxford University Press, 1977).

Dummett, Michael, Truth and other enigmas, (Duckworth, London, 1978).

Fourman, Michael P., The logic of Topoi, in Handbook of Math. Logic (ed. Barwise, J.), (North-Holland, 1977), p.1053-90.

Fourman, Michael P., Notions of Choice Sequence, Proc. Brouwer Symposium, (ed. Troelstra, A. and van Dalen, D.), (North-Holland, 1982).

Fourman, Michael P. \& Grayson, Robin J., Formal Spaces, Proc. Brouwer Symposium, (ed. Troelstra, A. and van Dalen, D.), (North-Holland, 1982).

Fourman, Michael P., T 1 spaces over topological sites, JPAA, (to appear), 1983. 
Freyd, Peter, Aspects of Topoi, Bul1. Austra1. Math. Soc., 7(1972), p.1-76.

Isbel1, John, Atomless parts of spaces, Math. Scand., 31(1972), p.5-32.

Johnstone, Peter T., Topos Theory, (Acad. Press, London, 1977).

Johnstone, Peter T., Stone spaces, (Acad. Press, London, 1982).

Joyal, André, \& Tierney, Myles, An extension of the Galois theory of Grothendieck, preprint, 1982.

Kreise], Georg, Lawless sequences of natural numbers. Comp. Math. 20(1968), p.222-48.

Makkai, Michael \& Reyes, Gonzalo, First-Order Categorical Logic, (Lecture Notes in Math. 611, Springer-Verlag, 1977).

Moschovakis, Joan R., A topological interpretation of second-order intuitionistic arithmetic, Comp. Math., (3), 26(1973), p.261-75.

Scott, Dana S., Extending the topological interpretation to intuitionistic analysis, Comp. Math. 20(1968), 222-48.

Scott, Dana S., Identity and Existence in Intuitionistic Logic, in Proc. Durham Symposium, (ed. Fourman et al.) (Lecture Notes in Math. 753, Springer-Verlag, 1978), p.660-96.

Troe1stra, Anne S., Choice Sequences, (Oxford University Press, 1977).

Wraith, Gavin C., Lectures on elementary topoi, Model theory and topoi, (ed. Lawvere F.W. et al.), (Lecture Notes in Math. 445, Springer-Verlag, Berlin, 1975), p.114-206.

Wright, Crispin, Wittgenstein's Philosophy of Mathematics, (Duckworth, 1981). 